\title{
Can Anorectal Manometry Findings Predict Subsequent Late Gastrointestinal Radiation Toxicity in Prostate Cancer Patients?
}

\author{
Yunseon Choi, MD ${ }^{1,2}$ \\ Won Park, MD, PhD² \\ Poong-Lyul Rhee, MD, PhD ${ }^{3}$
}

\section{${ }^{1}$ Department of Radiation Oncology,} Inje University Busan Paik Hospital, Inje University College of Medicine, Busan, Departments of ${ }^{2}$ Radiation Oncology and ${ }^{3}$ Internal Medicine, Samsung Medical Center, Sungkyunkwan University

School of Medicine, Seoul, Korea
Correspondence: Won Park, MD, PhD

Department of Radiation Oncology,

Samsung Medical Center,

Sungkyunkwan University School of Medicine,

81 Irwon-ro, Gangnam-gu, Seoul 06351, Korea

Tel: 82-2-3410-2600

Fax: 82-2-3410-2619

E-mail: wonro.park@samsung.com

Received November 24, 2014

Accepted January 16, 2015

Published Online March 13, 2015

\section{Purpose}

The purpose of this study is to investigate the influence of radiotherapy (RT) on anorectal function and radiation-induced toxicity in patients with prostate cancer.

\section{Materials and Methods}

Fifty-four patients who were treated with RT for prostate cancer (T1C-4NO-1M0) were evaluated. To assess the changes in anorectal function, two consecutive anorectal manometry readings were performed in patients, before and after 4-6 months of RT. Late gastrointestinal (GI) toxicity was defined as symptoms occurring more than 6 months after RT. The median radiation dose was 70.0 Gy (range, 66.0 to $74.0 \mathrm{~Gy}$ ). Whole pelvis field RT was performed in 16 patients (29.6\%). Grade of late radiation toxicity was defined in accordance to the severity of symptoms (Gulliford's scoring system).

\section{Results}

The median follow-up period was 60 months. Resting anal pressure $(p=0.001)$, squeeze pressure $(p<0.001)$, and urge to defecate volume $(p=0.025)$ were significantly reduced after RT. Fourteen patients (25.9\%) experienced late Gl toxicities. Among them, nine (16.7\%) showed severe (grade $\geq 2$ ) late toxicities. Elevated resting and squeeze external anal sphincter pressure prior to RT and large urge to defecate volumes after RT were associated with the occurrence of late $\mathrm{Gl}$ toxicities.

\section{Conclusion}

RT caused symptomatic anorectal dysfunction and resulted in a weakened anal sphincter. Increased urge to defecate volumes after RT were related to late GI toxicities. Elevated resting and squeeze anal sphincter pressure prior to RT rodcan be used to identify patients with an increased risk of late GI toxicities.

\section{Introduction}

Anorectal manometry (ARM) is commonly used to test anorectal physiology, and it is also used in studies of the anorectal toxicities after radiotherapy (RT) [1,2]; it could potentially be used to determine sensory functions and anorectal contractility.

The pathophysiology of radiation-induced anorectal dysfunction is still unclear. However, fibrosis seems to be
Key words

Prostatic neoplasms, Radiation toxicity, Anorectal manometry 
Table 1. Patient and treatment characteristics $(n=54)$

\begin{tabular}{lc} 
Variable & No. $(\%)$ \\
Median (range, yr) & $72(49-82)$ \\
ECOG performance status & \\
0 & $23(42.6)$ \\
1 & $31(57.4)$ \\
T stage & \\
T1c & $1(1.9)$ \\
T2 & $20(37.0)$ \\
T3a & $15(27.8)$ \\
T3b & $16(29.6)$ \\
T4 & $2(3.7)$ \\
N stage & \\
N0 & $38(70.4)$ \\
N1 & $16(29.6)$ \\
Gleason score & $7(6-10)$ \\
Median initial PSA (range, ng/mL) & $16.1(4.2-322.0)$ \\
Hormone therapy & $19(35.2)$ \\
Yes & $35(64.8)$ \\
No & \\
Radiotherapy & $70(66.0-74.0)$ \\
Median dose (range, Gy) & \\
RT volume & $16(29.6)$ \\
Whole pelvis & $38(70.4)$ \\
Prostate \pm SV & \\
Treatment technique & $40(74.1)$ \\
3D-CRT & $14(25.9)$ \\
IMRT & \\
\hline
\end{tabular}

ECOG, Eastern Cooperative Oncology Group; PSA, prostate-specific antigen; RT, radiotherapy; SV, seminal vesicle; 3D-CRT, 3-dimensional conformal radiotherapy; IMRT, intensity-modulated radiotherapy.

prostate cancer patients undergoing RT [8]. Prostate cancer patients treated with RT often complain about newly developed gastrointestinal (GI) symptoms, such as frequency, urgency, tenesmus, incontinence, and rectal bleeding [9]. Fecal incontinence [6] and rectal bleeding [10] are wellknown severe late complications of RT that can persist longterm. Fecal incontinence decreases the quality of the patients' lives over time [11]. In this study, we compared the results of ARM before and after RT to evaluate the patterns of anorectal function changes after RT used to treat prostate cancer. Also, we studied the relationship between the findings of ARM and late GI toxicities after RT in order to identify which patients are at increased risk for toxicities. This research is a new attempt to determine whether ARM findings are able to predict subsequent external beam RT late effects.

\section{Materials and Methods}

\section{Patients}

Fifty-four patients with prostate cancer were treated with definitive RT at Samsung Medical Center between 2007 and 2010. After approval by the Institutional Review Board (IRB File No. 2013-11-022), the medical and RT records of patients were retrospectively reviewed. All patients underwent ARM within 1 month before RT (pre-RT) and 4-6 months after RT (post-RT). The patients' characteristics are summarized in Table 1 . The median age of patients was 72 years (range, 49 to 82 years). Suspected pelvic lymph node metastasis was noted in 16 patients $(29.6 \%)$. Nineteen patients $(35.2 \%)$ underwent hormone therapy with RT.

\section{Radiation treatments}

All patients underwent simulation with computed tomography (CT) scanning for RT planning. A rectal catheter was inserted, and the balloon was inflated with $60 \mathrm{~cm}^{3}$ of air. For 40 patients, three-dimensional conformal radiotherapy (3D-CRT) was delivered. Intensity-modulated radiotherapy (IMRT) was used for 14 patients. The clinical target volume (CTV) consisted of the prostate and the adjacent seminal vesicle. Pelvic lymph nodes were delineated as CTV in 16 patients with suspected pelvic lymph node metastases. For organ at risk, rectal volume was generated as the rectal wall without lumen for the treatment planning. For 40 patients, the total RT dose was 66-74 Gy with 2 Gy per fraction, using 3D-CRT with 10-15 megavoltage photons. IMRT $(\mathrm{n}=14)$ was delivered with a total of $70 \mathrm{~Gy}$ in 28 fractions of $2.5 \mathrm{~Gy}$.

For 3D-CRT, the beam margins from CTV were $1.2 \mathrm{~cm}$ in all directions. For IMRT, the planning target volume (PTV) margins from CTV were $0.5 \mathrm{~cm}$, except for a posterior margin of $0.3-0.5 \mathrm{~cm}$. Daily image guidance with cone-beam CT and set-error correction for IMRT could aid in reduction of PTV margin compared to 3D-CRT.

For IMRT planning, the rectal dose constraints used in the study were as follows: the maximum rectal dose was $<74 \mathrm{~Gy}$, and $\leq 7 \%, \leq 20 \%, \leq 50 \%$, and $\leq 90 \%$ of the rectal volume received doses $>70 \mathrm{~Gy},>50 \mathrm{~Gy},>25 \mathrm{~Gy}$, and $>12 \mathrm{~Gy}$, respectively. However, no specific dose constraints were applied for the anus. For patients who underwent IMRT, image-guided RT was performed by using a megavoltage $\mathrm{CT}$ in helical tomotherapy.

\section{Anorectal manometry}

ARM was used to evaluate the anorectal function in this study. An 8-lumen polyvinyl recording assembly with eight 
Table 2. Changes in the parameters of anorectal manometry in patients before and after radiotherapy evaluated with a paired $t$ test

\begin{tabular}{lcrrr} 
Variable & Pre-RT & Post-RT & Pre-RT-Post-RT $(\Delta$ mean $)$ & p-value \\
Resting anal pressure (mm Hg) & $104.0 \pm 61.0$ & $82.2 \pm 41.1$ & 21.8 & 0.001 \\
Squeeze anal pressure (mm Hg) & $181.5 \pm 82.5$ & $142.8 \pm 57.6$ & 38.7 & $<0.001$ \\
Perception threshold volume (mL) & $24.4 \pm 9.6$ & $23.7 \pm 10.3$ & 0.7 & 0.376 \\
Urge to defecate volume (mL) & $195.4 \pm 58.9$ & $181.5 \pm 51.1$ & 13.9 & 0.025 \\
Anal canal length (cm) & $3.6 \pm 0.6$ & $3.6 \pm 0.6$ & 0.4 & 0.411 \\
IASP (mm Hg) & $54.3 \pm 18.2$ & $53.9 \pm 18.2$ & 1.2 & 0.939 \\
Resting EASP (mm Hg) & $88.8 \pm 35.6$ & $87.6 \pm 39.4$ & 4.1 & 0.824 \\
Maximum EASP (mm Hg) & $198.2 \pm 90.4$ & $194.1 \pm 83.7$ & 0.619 \\
\hline
\end{tabular}

Values are presented as mean \pm standard deviation. RT, radiotherapy; IASP, internal anal sphincter pressure; EASP, external anal sphincter pressure. a)Evaluation was performed only in 38 patients due to missing values.

side hole recording sites was initially used, and subsequently, a catheter with a distensible rectal balloon at the distal end was used. The side-hole recording sites were $0.5 \mathrm{~cm}$ apart in a spiral configuration around the tube. The anal sphincter pressure profile was obtained at each radial orientation by a slow pull-through of the sphincter. The recording assembly was then positioned across the sphincter to allow for continuous monitoring of internal and external sphincter activity. Patients were asked to voluntarily contract the sphincter as hard as possible in order to quantify the rectal balloon volume and anal sphincter tones. The balloon volumes associated with internal/external anal sphincter contraction and a sensation of rectal distention were noted.

Anal sphincter tone, functional anal canal length, and the sensation of rectal distention were assessed by ARM [12]. Resting anal sphincter pressure (ASP), defined as the difference between the intrarectal pressure and highest recorded ASP at rest, is generally recorded $1-2 \mathrm{~cm}$ from the anal verge. The maximum squeeze pressure was defined as the highest pressure that is recorded at any level within the anal canal during squeeze maneuver. The perception threshold volume was defined as the balloon volume that correlates with the first rectal distention. The urge to defecate volume was defined as the balloon volume at the moment when the patient first noted an urge to defecate. The anal canal length was defined as the length of the anal canal, over which the resting pressure exceeded that of the rectum by greater than $5 \mathrm{~mm} \mathrm{Hg}$ or, alternatively, as the length of the anal canal over which pressures were greater than half of the maximal pressure at rest. Internal ASP (IASP) was defined as the resting tone of the internal anal sphincter. Resting and maximum external ASP (EASP) were defined as the tones of the external anal sphincter during rest and during maximum voluntary contraction/ effort (in response to increased intra-abdominal pressure), respectively.

\section{Symptom evaluation}

The symptoms were assessed and recorded at every follow-up visit. Late GI toxicity was defined as symptoms occurring more than 6 months after RT. The degree of late GI tocixity was assessed in accordance to the late effects in normal tissues measured by using the new late radiation morbidity scoring schema by Gulliford et al. [13]. In patients with multiple clinical symptoms, multiple checking was allowed in symptom evaluation.

\section{Statistics}

The changes in the parameters of ARM before and after RT were evaluated with a paired $t$ test. The chi-square test was used to evaluate the relationship between RT-related toxicities and clinical factors (i.e., T stage) or treatment methods (i.e., hormone therapy, RT volume, and RT modality). Statistical correlation between late GI toxicities and anorectal manometric findings was tested by a binary logistic regression. The $p$-values of $<0.05$ (2-sided test) were considered to be statistically significant. Statistical analyses were performed using SPSS ver. 20 (IBM Co., Armonk, NY).

\section{Results}

\section{Functional changes}

Anal pressures at rest and in response to squeezing were significantly decreased after RT ( $\mathrm{p}=0.001$ and $\mathrm{p}<0.001$, respectively) (Table 2 ). The volumes at perception of rectal distention were not changed after RT. However, an urge to 
Table 3. Gastrointestinal toxicities after radiotherapy

\begin{tabular}{lccc} 
Symptom & Acute toxicities $(\leq 6 \mathrm{mo})$ & Late toxicities $(>6$ mo $)$ & Grade $\geq 2$ late toxicities ${ }^{\mathbf{a}}$ \\
\hline Rectal bleeding & $1(1.9)$ & $5(9.3)$ & $4(7.4)$ \\
Proctitis & $1(1.9)$ & $1(1.9)$ & 0 \\
Stool frequency & $1(1.9)$ & $1(1.9)$ & 0 \\
Sphincter control & $5(9.3)$ & $5(9.3)$ & $3(5.6)$ \\
Loose stools & $1(1.9)$ & $1(1.9)$ & $1(1.9)$ \\
Rectal urgency & $3(5.6)$ & $5(9.3)$ & $3(5.6)$ \\
Total patients & $8(14.8)$ & $14(25.9)$ & $9(16.7)$ \\
\hline
\end{tabular}

Values are presented as number (\%). a)Using late radiation morbidity scoring schema reported by Gulliford et al. [13]. Multiple checking of clinical symptoms was allowed in evaluation.

Table 4. Statistical correlations between late gastrointestinal toxicities $(n=14)$ and anorectal manometric findings using binary logistic regression

\begin{tabular}{lccc} 
& & p-value \\
\cline { 2 - 4 } Variable & Pre-RT & Post-RT & Pre-RT-Post-RT \\
Resting anal pressure & 0.039 & 0.826 & 0.795 \\
Squeeze anal pressure & 0.039 & 0.826 & 0.398 \\
Perception threshold volume & 0.999 & $>0.999$ & 0.807 \\
Urge to defecate volume & 0.051 & 0.029 & 0.753 \\
Mean anal canal length & 0.728 & 0.164 & 0.505 \\
IASPa) & 0.937 & 0.221 & 0.614 \\
Resting EASP & 0.099 & 0.842 & 0.195 \\
Maximum EASP & 0.104 & 0.380 & 0.518 \\
\hline
\end{tabular}

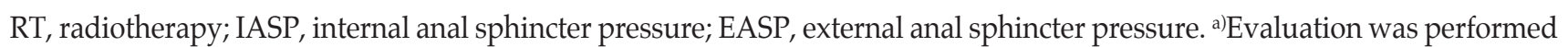
only in 38 patients due to missing values.

defecate volumes was significantly reduced after RT $(\mathrm{p}=0.025)$. The IASP and EASP were not significantly changed according to the implementation of RT.

\section{GI symptoms after RT}

The median follow-up period was 60 months (range, 7 to 73 months). Fourteen patients (25.9\%) showed late GI symptoms towards the end of RT (Table 3). Among them, sphincter control problems (including problems with subjective sphincter control and management sphincter control, $n=5$ ), and rectal urgency $(n=5)$ were common late toxicities (Table 3). Grade $\geq 2$ late GI toxicities were present in nine patients $(16.7 \%)$.

\section{Correlation between late GI toxicities and anorectal manometric findings}

Elevated pre-RT resting and squeezing ASP was associated with the occurrence of late GI toxicities ( $\mathrm{p}=0.039$ and $\mathrm{p}=0.039$, respectively) (Table 4). The mean pre-RT resting and squeezing ASP of patients who experienced late GI toxicities were $122.0 \mathrm{~mm} \mathrm{Hg}$ (range, 26.0 to $234.0 \mathrm{~mm} \mathrm{Hg}$ ) and $206.2 \mathrm{~mm} \mathrm{Hg}$ (range, 55.0 to $352.0 \mathrm{~mm} \mathrm{Hg}$ ), respectively. In contrast, the mean pre-RT resting and squeezing ASP of patients who did not experience late GI toxicities were $97.8 \mathrm{~mm} \mathrm{Hg}$ (range, 18.5 to $282.5 \mathrm{~mm} \mathrm{Hg}$ ) and $172.9 \mathrm{~mm} \mathrm{Hg}$ (range, 53.5 to $407.0 \mathrm{~mm}$ $\mathrm{Hg}$ ), respectively. In addition, the pre-RT urge to defecate volume tended to be associated with late GI toxicities $(p=0.051)$. Larger post-RT urge to defecate volumes were related to the occurrence of late GI toxicities $(\mathrm{p}=0.029)$ (Table 4). The mean post-RT urge to defecate volume of patients with late toxicities was $202.9 \mathrm{~mm} \mathrm{Hg}$ (range, 110.0 to 300.0 $\mathrm{mm} \mathrm{Hg}$ ). On the contrary, the mean post-RT urge to defecate volume of patients without late toxicities was $174.0 \mathrm{~mm} \mathrm{Hg}$ (range, 100.0 to $300.0 \mathrm{~mm} \mathrm{Hg}$ ). However, the differences between pre-RT ARM findings and post-RT ARM findings were not associated with late toxicities. Also, there was no specific correlation between grade $\geq 2$ late GI toxicities and ARM findings. 
There was no other clinical factors, except ARM findings, that significantly affected late GI toxicities. The $\mathrm{T}$ stage (T1-T2 vs. T3-T4) has not revealed any significant difference in the occurrence of late GI toxicity $(\mathrm{p}=0.202)$. Patients who were given hormone therapy $(\mathrm{n}=19)$ did not show higher incidence of late toxicity ( $\mathrm{p}=0.102)$. Both $\mathrm{RT}$ volume and RT modality (3D-CRT vs. IMRT) were not significantly associated with higher rate of late toxicity ( $p=0.308$ vs. $p=0.739$, respectively).

\section{Discussion}

Preventing a treatment-related complication is as important as finding a cure for the disease. By using ARM, we were able to assess functional changes related to RT and to predict which patients are at risk for radiation-induced toxicities.

The pathologic mechanism of RT-induced GI toxicities is still unclear. RT induces fibrosis in the anorectal wall and anal sphincter [14]. An increased amount of collagen in the muscular layer of the rectum and internal anal sphincter is reported to be associated with fecal incontinence [5]. Decreased sensitivity to rectal distension (urge to defecate volume) was observed in patients with late GI toxicities in accordance to the results of this study. Inflammation and fibrotic changes, as well as collagen deposition might have occurred after irradiation, and it might be related to functional and structural changes of anorectum. Incoordination in sensory and motor response of anal sphincter, such as increased urge to defecate volumes, and may bring on chronic rectal mucosa damage in the way of stool passage. Altered neuronal structure or chemical mediators of inflammation might also influence rectal sensitivity. The majority of patients with late radiation proctitis showed anorectal sensory/motor dysfunction [14]. Further research is needed to clarify the mechanism of RT-induced GI toxicities.

RT for prostate cancer can cause severe chronic anorectal dysfunction, which is the result of a weakened anal sphincter [15]. Our data also showed that anal pressure at rest and in response to squeezing were reduced after RT. In addition, the level of resting and squeezing anal pressure may be used to predict which patients will experience late GI toxicities. Urge to defecate volumes, which significantly reduced according to RT, were associated with abnormal clinical symptoms after RT. In contrast to the previous study by Yeoh et al. [5], the perception threshold was not significantly changed after RT in our study. Patients with subclinical dysfunction before RT, such as the elevated ASP in this study, are more vulnerable to late GI toxicities. Elevated ASP could make it difficult to deal with the ordinary intraluminal volumetric changes of anorectum, such as stool passage. Severe constipation, caused by elevated ASP, may evoke objective complications, such as perianal mucosal damage or anal sphincter problems. Therefore, higher ASP may bring on late GI toxicities. According to our data, elevated pre-RT ASP appears to be predictive of late GI toxicities; it might be able to be utilized for patient selection for RT.

In our study, late GI toxicity was not significantly related to the RT volume or to the modality used. However, the occurrence of GI toxicity is known to be related to the radiation dose to the anorectal wall [7] and external anal sphincter. Michalski et al. [8] reported that a large rectal volume (> 15\%) that receives more than $70 \mathrm{~Gy}$ was significantly associated with grade $\geq 2$ rectal toxicity. Therefore, it seems to be reasonable to consider using an IMRT technique, which excludes the external anal sphincter and anorectal wall. Dosimetric consideration to rectal wall seems feasible to reduce the risk of late GI toxicity. Krol et al. [16] argued that sparing the structures related to fecal continence from irradiation is crucial to reduce late GI dysfunction. In this regard, using a hydrogel spacer between the prostate and rectum seems feasible to prevent a radiation-induced rectal toxicity by reducing the irradiation dose to the rectum. In patients predicted to have late GI toxicities based on pre-RT ARM, placing spacers may be helpful to reduce late toxicity $[16,17]$.

Our study has several limitations; as it is based on ARM findings, it does not include an evaluation of anatomic anorectal changes after RT. Different symptoms might originate from different anatomical structures that are damaged by RT. Smeenk et al. [2] demonstrated that urgency and incontinence were associated with the dose of RT delivered to both the anus and rectum, whereas frequency was associated with the dose of RT delivered to the anus. Separate organ drawings (i.e., the anus) in an RT plan might be helpful for anatomical evaluation. This study was designed to detect intraindividual changes over time. Therefore, interindividual variations, such as body mass index, age, or medical comorbidities could indirectly affect the ARM results [18]. In addition, our study results were based on a small number of patients. Therefore, heterogenous composition of patients may have influenced the results. Our study should be verified through large-scale prospective studies. Moreover, functional changes may occur over time after RT. Further long-term follow-up with ARM could provide valuable data related to the time-course of functional changes.

Further large-scale cohort studies are warranted to understand the mechanism of radiation-induced anorectal dysfunction. Recently introduced 3-dimensional high-definition ARM could provide a 3-dimensional pressure gradient and topographical information. Electromyography and nerve conduction tests or endoscopy might be useful to 
inspect structural damage [8]. A well-designed dosimetric analysis of dose-volume parameters in the anorectum is needed to provide a more precise mechanism. It was previously reported that a reduced rectal wall surface exposed to radiation results in less mucosal changes and rectal toxicity [19]. The quality of life after RT for prostate cancer is largely affected by anorectal radiation toxicity, such as urgency or frequency $[7,20]$. Therefore, question-based, patient-reported quality of life analyses could contribute to understanding toxicities.

\section{Conclusion}

RT caused symptomatic anorectal dysfunction. An increased anal pressure prior to RT and urge to defecate volume after RT was related to an occurrence of late GI toxicities. Application of ARM for screening patients who have an elevated ASP prior to RT could be helpful in identifying patients with an increased risk of late GI toxicities.

\section{Conflicts of Interest}

Conflict of interest relevant to this article was not reported.

\section{References}

1. Yeoh EK, Holloway RH, Fraser RJ, Botten R, Di Matteo A, Moore JW, et al. Anorectal function after three- versus twodimensional radiation therapy for carcinoma of the prostate. Int J Radiat Oncol Biol Phys. 2009;73:46-52.

2. Smeenk RJ, Hopman WP, Hoffmann AL, van Lin EN, Kaanders JH. Differences in radiation dosimetry and anorectal function testing imply that anorectal symptoms may arise from different anatomic substrates. Int J Radiat Oncol Biol Phys. 2012;82:145-52.

3. Mantini G, Tagliaferri L, Mattiucci GC, Balducci M, Frascino V, Dinapoli N, et al. Effect of whole pelvic radiotherapy for patients with locally advanced prostate cancer treated with radiotherapy and long-term androgen deprivation therapy. Int J Radiat Oncol Biol Phys. 2011;81:e721-6.

4. Yeoh EK, Bartholomeusz DL, Holloway RH, Fraser RJ, Botten R, Di Matteo A, et al. Disturbed colonic motility contributes to anorectal symptoms and dysfunction after radiotherapy for carcinoma of the prostate. Int J Radiat Oncol Biol Phys. 2010; 78:773-80.

5. Yeoh EK, Holloway RH, Fraser RJ, Botten RJ, Di Matteo AC, Butters J. Pathophysiology and natural history of anorectal sequelae following radiation therapy for carcinoma of the prostate. Int J Radiat Oncol Biol Phys. 2012;84:e593-9.

6. Maeda Y, Hoyer M, Lundby L, Norton C. Faecal incontinence following radiotherapy for prostate cancer: a systematic review. Radiother Oncol. 2011;98:145-53.

7. Krol R, Hopman WP, Smeenk RJ, Van Lin EN. Increased rectal wall stiffness after prostate radiotherapy: relation with fecal urgency. Neurogastroenterol Motil. 2012;24:339-e166.

8. Michalski JM, Gay H, Jackson A, Tucker SL, Deasy JO. Radiation dose-volume effects in radiation-induced rectal injury. Int J Radiat Oncol Biol Phys. 2010;76(3 Suppl):S123-9.

9. Berndtsson I, Lennernas B, Hulten L. Anorectal function after modern conformal radiation therapy for prostate cancer: a pilot study. Tech Coloproctol. 2002;6:101-4.

10. Kim GE, Lim JJ, Park W, Park HC, Chung EJ, Seong J, et al. Sensory and motor dysfunction assessed by anorectal manometry in uterine cervical carcinoma patients with radiationinduced late rectal complication. Int J Radiat Oncol Biol Phys. 1998;41:835-41.

11. Bartlett L, Nowak M, Ho YH. Impact of fecal incontinence on quality of life. World J Gastroenterol. 2009;15:3276-82.

12. Kim JH. How to interpret conventional anorectal manometry. J Neurogastroenterol Motil. 2010;16:437-9.

13. Gulliford SL, Foo K, Morgan RC, Aird EG, Bidmead AM, Critchley $\mathrm{H}$, et al. Dose-volume constraints to reduce rectal side effects from prostate radiotherapy: evidence from MRC RT01 Trial ISRCTN 47772397. Int J Radiat Oncol Biol Phys. 2010;76:747-54.

14. Andreyev J. Gastrointestinal complications of pelvic radiotherapy: are they of any importance? Gut. 2005;54:1051-4.

15. Lundby L, Krogh K, Jensen VJ, Gandrup P, Qvist N, Overgaard $\mathrm{J}$, et al. Long-term anorectal dysfunction after postoperative radiotherapy for rectal cancer. Dis Colon Rectum. 2005;48:1343-9.

16. Krol R, Smeenk RJ, van Lin EN, Hopman WP. Impact of late anorectal dysfunction on quality of life after pelvic radiotherapy. Int J Colorectal Dis. 2013;28:519-26.

17. Pinkawa M. Spacer application for prostate cancer radiation therapy. Future Oncol. 2014;10:851-64.

18. Longobardi B, Berardi G, Fiorino C, Alongi F, Cozzarini C, Deli A, et al. Anatomical and clinical predictors of acute bowel toxicity in whole pelvis irradiation for prostate cancer with Tomotherapy. Radiother Oncol. 2011;101:460-4.

19. van Lin EN, Kristinsson J, Philippens ME, de Jong DJ, van der Vight LP, Kaanders JH, et al. Reduced late rectal mucosal 
changes after prostate three-dimensional conformal radiotherapy with endorectal balloon as observed in repeated endoscopy. Int J Radiat Oncol Biol Phys. 2007;67:799-811.

20. Bacon CG, Giovannucci E, Testa M, Glass TA, Kawachi I. The association of treatment-related symptoms with quality-of-life outcomes for localized prostate carcinoma patients. Cancer. 2002;94:862-71. 